\title{
The Development and Application of One Thermal-Hydraulic Program Based on ANSYS for Design of Ceramic Breeder Blanket of CFETR
}

\author{
Kecheng Jiang ${ }^{1,2} \cdot{\mathrm{Jia} \mathrm{Li}^{2} \cdot \text { Xiaokang Zhang }}^{1,2} \cdot$ Songlin Liu ${ }^{1}$
}

Published online: 11 April 2015

(C) The Author(s) 2015. This article is published with open access at Springerlink.com

\begin{abstract}
Thermal-hydraulic design and analysis is an important step for blanket design to determine the temperature of the material, hydraulics parameter and radial building, especially in scope optimization phase. Traditionally, thermal-hydraulic calculation is a tedious work for designer while combining with neutronic design iteration calculation through ANSYS graphical user interface operation. One secondary development program was developed based on the commercial software ANSYS and characteristics of ceramic breeder layer-separated type blanket characteristics. When the material type, dimension and nuclear heat deposition of each layer along the blanket radial direction act as the input parameters, this program can automatically create geometric model, generate mesh, adding material properties, set boundary condition, calculate and post-process the calculation data according to user definition by calling the ICEM and Fluent software. Finally, the visual two-dimensional temperature field and the max temperature of each layer are obtained promptly. This could help easily to determine the thermal characteristics and hydraulics parameter of blanket and reduce the workload of the operators. In this paper, the programming methodology is reported and verified the reliability and efficiency of this program by employing one blanket design scheme for thermal-hydraulic calculation for Chinese Fusion Engineering Test Reactor.
\end{abstract}

Songlin Liu

slliu@ipp.ac.cn

1 Institute of Plasma Physics, Chinese Academy of Sciences, Hefei 230031, Anhui Province, China

2 University of Science and Technology of China, Hefei 230031, Anhui Province, China
Keywords Blanket - Thermal-hydraulic · ANSYS · Program

\section{Introduction}

Chinese Fusion Engineering Test Reactor (CFETR) [1] is an ITER-like superconducting tokamak reactor, which requires tritium self-sufficiency under $200 \mathrm{MW}$ fusion power. As one key component in the fusion reactor, the blanket has three main functions: (1) Producing tritium through the ${ }^{6} \mathrm{Li}(\mathrm{n}, \alpha) \mathrm{T}$ and ${ }^{7} \mathrm{Li}(\mathrm{n}, \alpha) \mathrm{T}$ reaction [2]; (2) Removing the nuclear heat deposition and the high heat flux from the plasma by the coolant flowing through the internal passages; (3) Providing shielding to protect the superconducting magnet from neutron radiation. The blanket could be classified as solid and liquid breeder blanket according to the tritium breeders, or be classified as water cooled, helium cooled and liquid metal cooled blanket according to the coolant. Tritium breeder and neutron multiplier are hierarchical arrangement generally for solid breeder blanket along the radial direction, also with the cooling plates inserted to remove the heat away.

The procedure of the blanket design is to carry out the neutron analysis based on the preliminary radial structure arrangement firstly, then change the tritium breeder, neutron multiplier and cooling plates arrangement to achieve the ideal tritium breeder ratio (TBR). Secondly, under the blanket structure established by neutron calculation, thermal-hydraulic calculation needs conducted to ensure that the max temperature of all materials in the blanket is below the limit and the breeding zone temperature is higher than tritium release temperature. Finally, structure stress analysis will be carried out to confirm the material stress not exceeding the limit. Therefore, iterative calculation 
between neutron, thermal-hydraulic and structure analysis is necessary to obtain the optimal structure. Thermal-hydraulic analysis is one important part of the blanket design, especially for one dimensional design phase to optimize the radial scope size and to accelerate the blanket research process. In order to numerically investigate the thermalhydraulic parameters in one-dimensional structure design phase, on the basis of ANSYS software, one secondary development program which could create geometric model, generate mesh, set boundary condition, calculate and postprocess the results automatically is developed, releasing the researchers from the heavy work through the traditional graphical user interface (GUI) operation.

In this paper, the programming methodology is introduced and verified the reliability and efficiency of this program by employing one kind of blanket thermal-hydraulic calculation for CFETR.

\section{Program Creation}

\section{Program Description}

In this program, the coolant flowing arrangement is assumed to be fixed, namely the coolant flows along the blanket poloidal direction from top to bottom to cool the first wall firstly, then flows through the whole blanket along the radial direction in series. The two-dimensional blanket structure diagram is given in Fig. 1, one color represents one kind of material, and the layers are accumulated along the radial direction. Each layer has its own material type, dimension and nuclear heat deposition. According to the characteristic of classes and objects encapsulating data in $\mathrm{C}++$ language [3], the blanket class which includes the material type, dimension and nuclear heat deposition is defined firstly, each layer is one object in the blanket class. All the objects are packed up by vector defined in the program, then the journal file [4] which records the procedure of GUI operation and is the core technology to

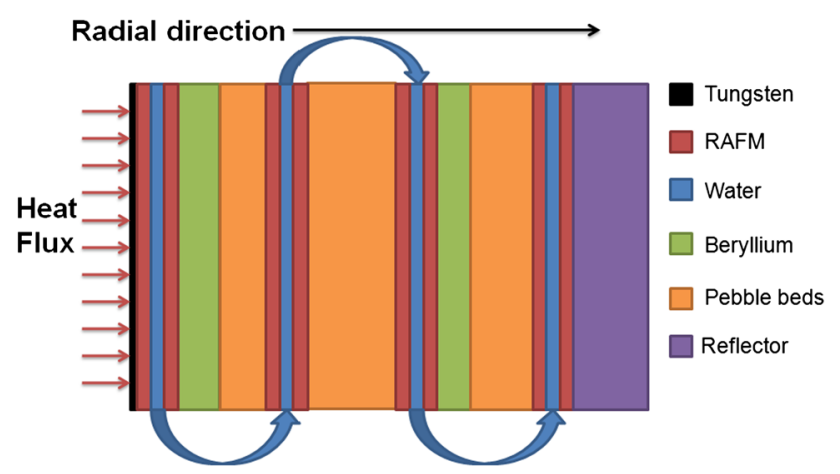

Fig. 1 The two-dimensional blanket structure support secondary development in ICEM and Fluent could be generated flexibly. Part of the journal file is given in Fig. 2. The program transfers the journal file to ICEM and Fluent successively by the calling code, given in Fig. 3, then the operation will run automatically without the tedious GUI operation. The program would output the visual two-dimensional temperature field and the max temperature of the each layer, helping the operators to estimate whether the structure temperature exceeds the allowable limit and then optimize the structure design in turn. The whole procedure of this program is given in Fig. 4.

In addition to the data including material type, dimension and nuclear heat deposition of each layer could be adjusted, there are other variables also can be altered, including coolant velocity, heat flux from plasma and even the height along poloidal direction of the blanket, adapting various blanket design scheme for CFETR.

\section{Materials Included in this Program}

According to the materials which the blanket employs, the relevant material library is build up in the program. In this library, coolant includes helium and water and the relationship that the specific heat, thermal conductivity and density vary with temperature changing is included in this program. Solid material includes tungsten as protection material for first wall, RAFM steel as structure material, tritium breeder and neutron multiplier in the elemental form including $\mathrm{Be}, \mathrm{Be}_{12} \mathrm{Ti}, \mathrm{Li}_{4} \mathrm{SiO}_{4}, \mathrm{Li}_{2} \mathrm{TiO}_{3}, \mathrm{Li}_{2} \mathrm{O}$ and $\mathrm{Li}_{2} \mathrm{ZrO}_{3}$ and tritium breeder with neutron multiplier in the pebble bed form including $\mathrm{Be}, \mathrm{Li}_{4} \mathrm{SiO}_{4} / \mathrm{He}, \mathrm{Li}_{2} \mathrm{ZrO}_{3} / \mathrm{He}$ and $\mathrm{Li}_{2} \mathrm{TiO}_{3} / \mathrm{Be}_{12} \mathrm{Ti} / \mathrm{He}$. The included thermal physical parameters in this program for pebble beds, including thermal conductivity and the volume ratio between breeder and helium of pebble beds, are all from the references [59] temporarily, giving the relationship that the thermal conductivity vary with temperature changing.

$\begin{array}{lllll}\text { vertex creat coordinates } & 0 & 0 & 0 \\ v e r t e x & \text { creat coordinates } & 2 & 0 & 0 \\ \text { vertex creat coordinates } & 5 & 0 & 0 \\ \text { vertex creat coordinates } & 13 & 0 & 0 \\ \text { vertex creat coordinates } & 16 & 0 & 0 \\ v e r t e x & \text { creat coordinates } & 51 & 0 & 0 \\ \text { vertex creat coordinates } & 53 & 0 & 0 \\ \text { vertex creat coordinates } & 58 & 0 & 0 \\ \text { vertex creat coordinates } & 60 & 0 & 0 \\ \text { vertex creat coordinates } & 110 & 0 & 0 \\ \text { vertex creat coordinates } & 112 & 0 & 0 \\ \text { vertex creat coordinates } & 117 & 0 & 0 \\ \text { vertex creat coordinates } & 119 & 0 & 0\end{array}$

Fig. 2 Journal file to create geometry model points 
CString cmdline("-vx 2d -i E:\\blanketcode $\backslash 21 \backslash \backslash f l u e n t . j o u ") ;$ CString m_FluentDir("D:\\Fluent.Inc6.2\|ntbin\\ntx86\\fluent.exe"); CString m_WorkDir("E:\|blanketcode\\21");

STARTUPINFO StartupInfo;

memset(\&Startuplnfo,0,sizeof(STARTUPINFO));

Startup Info.cb = sizeof(STARTUPINFO);

StartupInfo.dwFlags=STARTF_USESHOWWINDOW;

Startupinfo.wShowWindow=SW_HIDE;

PROCESS_INFORMATION Processinfo;

if(!::CreateProcess(m_FluentDir,cmdline.GetBuffer(cmdline.GetLength( I),

NULL,NULL,FALSE,O,NULL,m_WorkDir,\&StartupInfo,\&ProcessInfo))

\{cout<<"CreateFail!"<<endl;

return;\}

WaitForSingleObject(Process/nfo.hProcess,INFINITE);

Fig. 3 The code used to call ICEM and Fluent

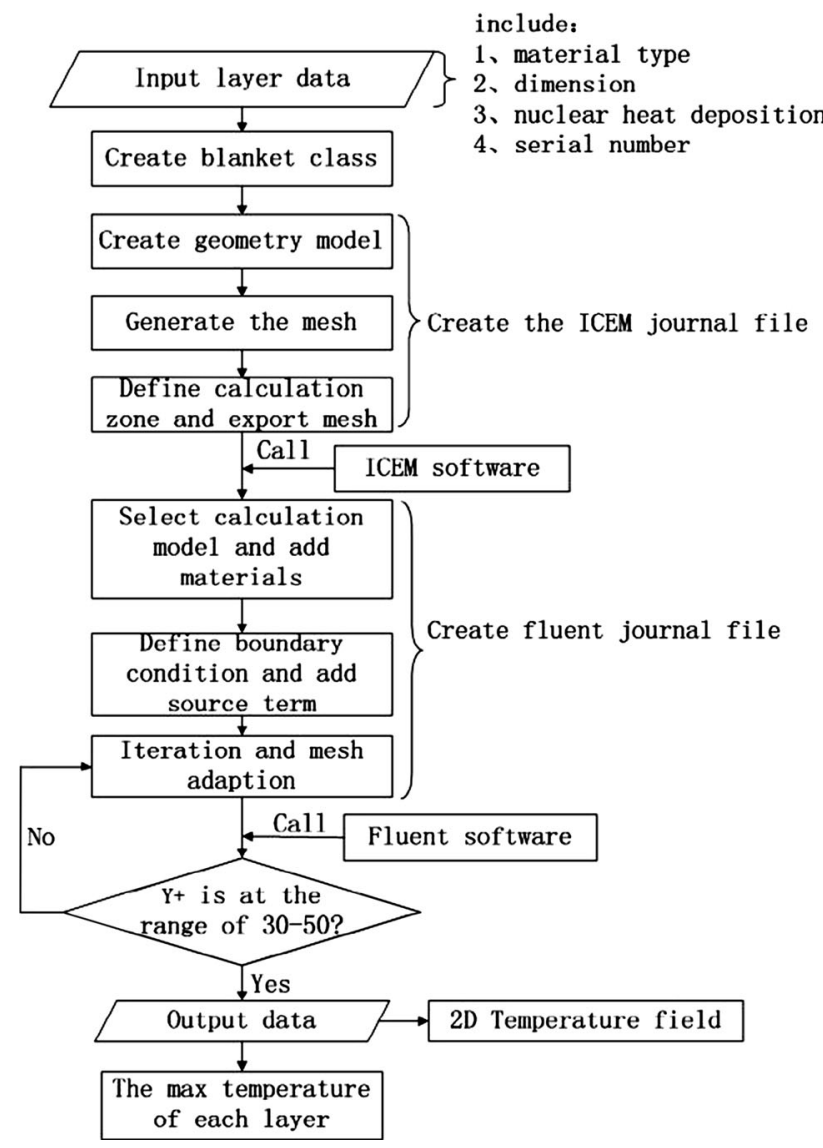

Fig. 4 The whole flow diagram of this program

\section{Meshing and Calculation Model Setting}

Considering the regularity characteristic of the two-dimensional fusion blanket structure, quadrilateral is used for meshing and the mesh size is set as one adjustable variable in the program. The passages in the blanket involve flowing heat transfer process, coolant velocity tends to be higher in order to remove the huge heat in time and the flowing model could be judged as turbulent through Reynolds number generally. The boundary layer near the channel wall has great impact on heat transfer, then the first mesh node should be ensured at the zone of logarithmic law, namely the distance named $\mathrm{Y}^{+}$between the center of first mesh and channel wall should be at the reasonable range, ensuring that the quantities of the zone near wall where molecular viscosity effects obviously could be connected with the quantities of the turbulent core zone by the semiempirical formulas in the turbulent calculation model. In order to satisfy this requirement, the mesh adaption process method [10] is adopted to deal with the zone near the channel wall in this program when calling Fluent. During the iteration calculation, adjusting the mesh size and mesh spacing timely to adapt the $\mathrm{Y}^{+}$to the range of 30-50 which is the requirement that the turbulent model needs. There is a judgment statement to repeat the mesh adaption process until the $\mathrm{Y}^{+}$satisfies the requirement in this program. When calling Fluent, the standard K- $\varepsilon$ turbulent model is chosen to close the Reynolds stress term to solve the momentum equation, the SIMPLE algorithm is used to realize the pressure and velocity coupling, a second-order upwind scheme is chosen for energy and momentum equations and the standard channel wall function is adopted to deal with the quantities in the viscous sub-layer.

During the mesh adaption process, with the purpose of obtaining the reasonable $\mathrm{Y}^{+}$as soon as possible, the following semi-empirical formulas (1,2 and 3) are adopted to forecast the value which is the distance between the first mesh point and channel wall before meshing, then the mesh arrangement approaching the ideal mesh near the channel wall is obtained, saving a lot of time at the mesh adaption process.

$\bar{c}_{f} / 2 \approx 0.039 \mathrm{Re}_{D}^{-0.2}$

$u_{\tau} \equiv \sqrt{\tau_{w} / \rho}=U_{e} \sqrt{\bar{c}_{f} / 2}$

$Y^{+}=50 v / u_{\tau}$

where $\bar{c}_{f}$ is the channel flow friction coefficient, $\operatorname{Re}_{\mathrm{D}}$ is the Reynolds number, $U_{e}$ is the main velocity $\mathrm{m} / \mathrm{s}$ and $v$ is the dynamic viscosity $\mathrm{m}^{2} / \mathrm{s}$.

\section{Program Verification}

In this paper, thermal-hydraulic calculation is made to a selected blanket structure design by this program, the data of each layer is the input parameters, finally output all results the program could generate, including the two-dimensional visual temperature field of whole blanket and each material, also with the max temperature of each layer, 
verifying the realizability and efficient performance of this program.

\section{The Input Data of Each Layer}

The material type, dimension and nuclear heat deposition of each layer along the blanket radial direction are the input parameters in this program, the detail data is given in Table 1. The one-dimensional nuclear heat deposition in the structure is calculated by MCNP. For the sake of the higher required TBR, more mixed pebble beds $\mathrm{Li}_{2} \mathrm{TiO}_{3} /$ $\mathrm{Be}_{12} \mathrm{Ti} / \mathrm{He}$ is arranged in the blanket to produce tritium. At the front of the blanket, there places the beryllium multiplying the neutron to supplement the neutron which is absorbed by the other materials that do not generate tritium. At the back of the blanket, the thick $\mathrm{ZrH}_{2}$ is placed to shield and reflect the neutron, protecting the environment and superconducting magnet from neutron radiation [11], at the meantime, also increasing the neutron availability and TBR effectively. The RAFM steel and coolant arrangement in the first wall and cooling plates is given in the Fig. 5.

For the thermal-hydraulic boundary condition setting in Fluent, the coolant water velocity is $1 \mathrm{~m} / \mathrm{s}$, the operating pressure is $15.5 \mathrm{MPa}$ [12], coolant inlet temperature is

Table 1 The input data of each layer along the radial direction

\begin{tabular}{lcl}
\hline Material type & Dimension $(\mathrm{mm})$ & Nuclear heat deposition $\left(\mathrm{MW} / \mathrm{m}^{3}\right)$ \\
\hline $\mathrm{Wu}$ & 2 & 6.63124 \\
$\mathrm{FW}$ & 14 & 1.07233 \\
$\mathrm{Be}_{1}$ & 35 & 1.23374 \\
$\mathrm{CP}_{1}$ & 9 & 0.81739 \\
$\mathrm{~B}_{1}$ & 50 & 1.21518 \\
$\mathrm{CP}_{2}$ & 9 & 0.95454 \\
$\mathrm{~B}_{2}$ & 50 & 0.81145 \\
$\mathrm{Be}_{2}$ & 20 & 0.43242 \\
$\mathrm{CP}_{3}$ & 9 & 0.57401 \\
$\mathrm{~B}_{3}$ & 70 & 0.44139 \\
$\mathrm{CP}_{4}$ & 9 & 0.35181 \\
$\mathrm{~B}_{4}$ & 60 & 0.24433 \\
$\mathrm{CP}_{5}$ & 9 & 0.22543 \\
$\mathrm{~B}_{5}$ & 50 & 0.15680 \\
$\mathrm{CP}_{6}$ & 9 & 0.15268 \\
$\mathrm{~B}_{6}$ & 40 & 0.10560 \\
$\mathrm{CP}_{7}$ & 9 & 0.11094 \\
$\mathrm{~B}_{7}$ & 29 & 0.24382 \\
$\mathrm{CP}_{8}$ & 9 & 0.09039 \\
$\mathrm{ZrH}_{2}$ & 100 & 0.05399 \\
\hline
\end{tabular}

$F W$ first wall, $C P$ cooling plate, $B$ the mixed $\mathrm{Li}_{2} \mathrm{TiO}_{3} / \mathrm{Be}_{12} \mathrm{Ti} / \mathrm{He}$ pebble beds

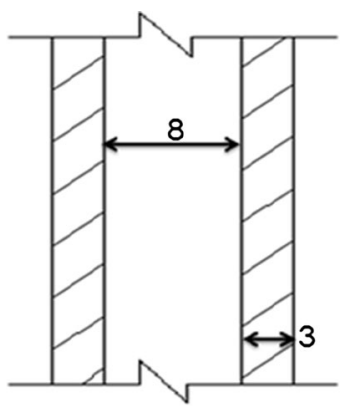

(a) FW

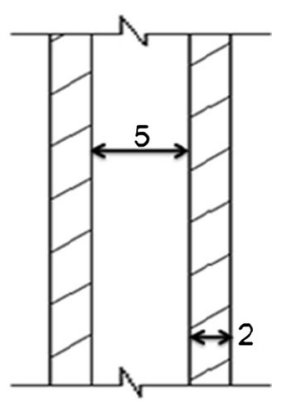

(b) $\mathrm{CP}$
Fig. 5 The RAFM steel and coolant arrangement in FW and cooling plate

$558.15 \mathrm{~K}$ and the heat flux from plasma on $\mathrm{FW}$ is set to be $1 \mathrm{MW} / \mathrm{m}^{2}$.

\section{Calculation Results Analysis}

The two-dimensional temperature field of the whole blanket and each kind of material is given in Figs. 6, 7, 8 and 9. Coolant water flows through the blanket along the blanket radial direction in series, as given in Fig. 6, the first wall withstands the huge heat flux of $1 \mathrm{MW} / \mathrm{m}^{2}$, then there appears the higher temperature filed at the FW zone. Figure 7 gives the temperature field of tritium breeder, the highest temperature appears in the front where the neutron heat flux is larger causing that the reaction of $\operatorname{Li}_{6}(n, \alpha) T$ which generates heat is more frequently, besides, the heat flux from plasma is higher. Figure 8 gives the temperature field of beryllium, because the FW is cooled by water first, then the lowest temperature is located at the front zone. At the back, coolant water temperature rises, causing heat transfer becomes worse, then temperature is more higher.

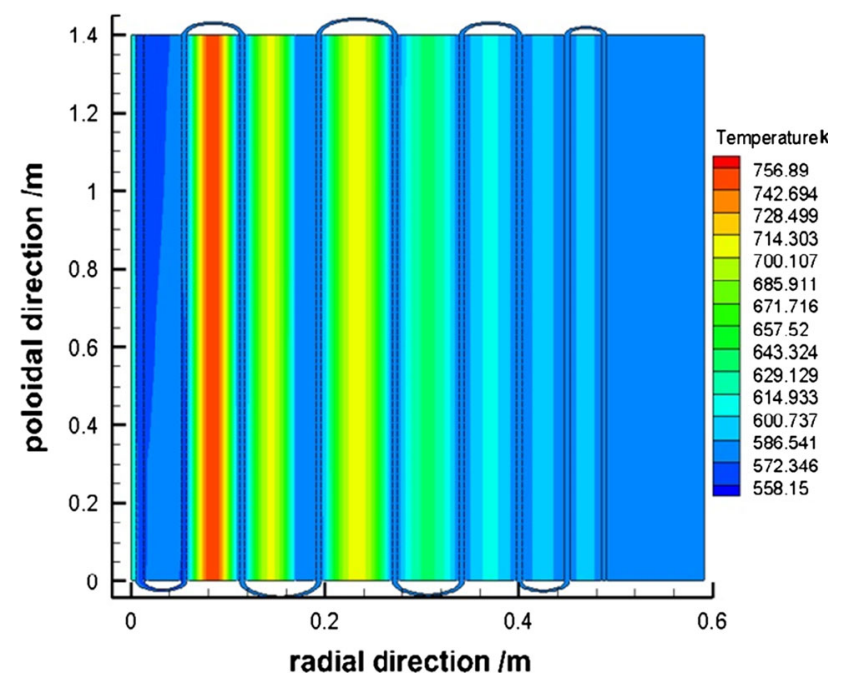

Fig. 6 Temperature distribution of the whole blanket 


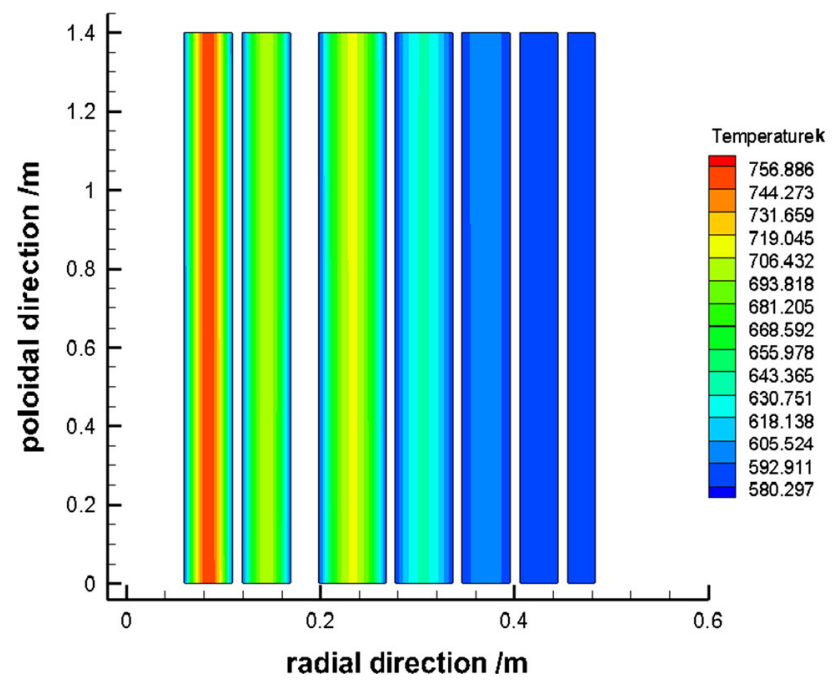

Fig. 7 Tritium breeding zones temperature distribution

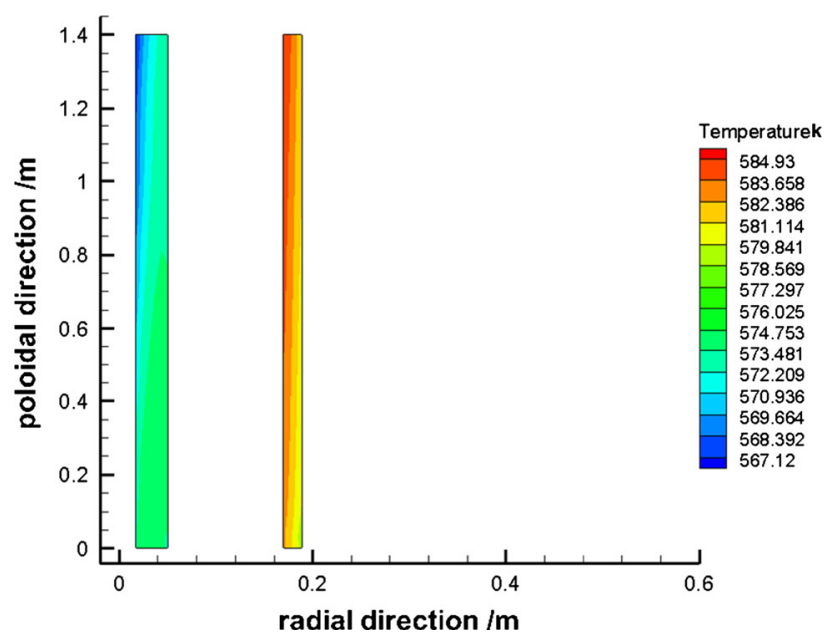

Fig. 8 Neutron multiplier zones temperature distribution

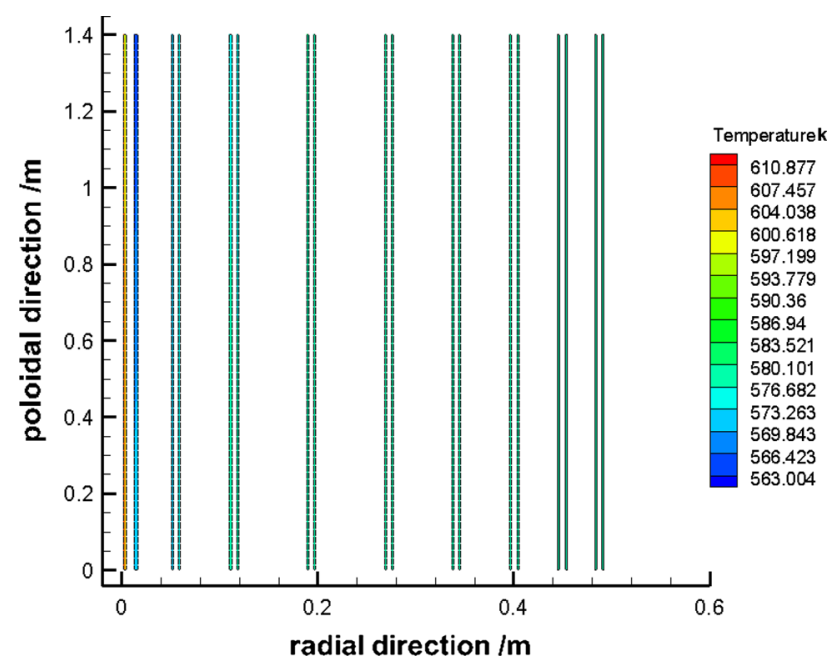

Fig. 9 RAFM steel zones temperature distribution
The hottest beryllium is located at the middle where the nuclear heat deposition on the adjacent sides where is the tritium breeder is more higher. Figure 9 gives the temperature field of RAFM steel, the hot point is at the front. In conclusion, the material temperature is the result of interaction of nuclear heat deposition and the heat transfer capacity of coolant. Table 2 gives the max temperature of each material obtained by dichotomization method in this program. Operators could judge whether the max temperature exceeds the allowable limit and then take measures to optimize the blanket structure, rearranging the arrangement of the cooling plate, neutron multiplier and tritium breeder. Taking the allowable temperature [13] given in the table as the design criteria, this selection blanket structure is safe enough from the perspective of thermal-hydraulic.

It takes about 23 min to get all the results by this program, however, it will take much more time about $57 \mathrm{~min}$ to get the same results through the traditional GUI operation and the efficiency increases by $59.6 \%$ through this program. Then, it can be concluded that this program does save a lot of time for blanket design and avoid much more unnecessary work.

\section{Conclusion}

There is the requirement of coupling calculation among neutronic, thermal-hydraulic and structure for fusion blanket design of CFETR, it needs repeating iteration calculation among the three aspects. However, huge work will be required for the thermal-hydraulic calculation through the GUI operation. This paper has developed the program which could create geometric model, generate mesh, set boundary condition, calculate and post-process the calculation data automatically based on ANSYS software with $\mathrm{C}++$ language which has the characteristic of classes and objects encapsulating data for thermal-hydraulic calculation. The material type, structure dimension and nuclear heat deposition of each layer are the input parameters in this program. The zone near channel wall which has the huge impact on coolant flow heat transfer is handled with the mesh adaption method, improving the

Table 2 The max temperature of each material

\begin{tabular}{llc}
\hline Material type & Max temperature $(\mathrm{K})$ & Allowable limit $(\mathrm{K})$ \\
\hline RAFM steel & 610.88 & 823.15 \\
Tungsten & 615 & 1573.15 \\
Pebble beds & 756.89 & 1173.15 \\
Beryllium & 584.93 & 873.15 \\
\hline
\end{tabular}


calculation accuracy. This program finally outputs the twodimensional temperature field also with the max temperature of each layer, providing for the operators to judge whether the blanket design is safe or not and then optimize the structure design. Thermal-hydraulic calculation is done for a selection blanket through this program, and the results shows that this program could increase calculation efficiency by $59.6 \%$ compared with the traditional GUI operation, reducing the workloads of the researcher's greatly.

In this program, the coolant flow arrangement is fixed. The improvement should be made to realize the coolant channel series or parallel arrangement flexibly in the purpose of adapting to the various coolant flow solutions in the future work.

Acknowledgments The authors wish to acknowledge the financial support of National special project for magnetic confined nuclear fusion energy with Grant Nos. 2013GB108004, 2015GB108002, 2014GB122000 and 2014GB119000, and the Chinese National Natural Science Foundation under Grant No. 11175207 for this research.

Open Access This article is distributed under the terms of the Creative Commons Attribution 4.0 International License (http:// creativecommons.org/licenses/by/4.0/), which permits unrestricted use, distribution, and reproduction in any medium, provided you give appropriate credit to the original author(s) and the source, provide a link to the Creative Commons license, and indicate if changes were made.

\section{References}

1. Wan Yuanxi, Design and strategy for the Chinese fusion engineering testing reactor (CFETR) (SOFT, San Francisco, 2013)
2. Ma. Xuebin, Liu Songlin, Li Jia et al., Preliminary design of a helium-cooled ceramic breeder blanket for CFETR based on BIT concept. Plasma Sci. Technol. 16, 390-395 (2014)

3. I. Horton, Beginning $C++$ (Tsinghua University Press, Beijing, 2008), pp. 45-56. (in Chinese)

4. Xiao Hong, Gao Chao, Dang Yunqing et al., Secondary development of FLUENT and application in numerical simulation of aerodynamic characteristic for rockets. Aeronaut. Comput. Tech. 39, 55-57 (2009). (in Chinese)

5. M. Uchida, E. Ishitsuka, H. Kawamura, Thermal conductivity of neutron irradiated Be $\mathrm{Be}_{12}$ Ti. Fusion Energ. Des. 69, 499-504 (2003)

6. Shigeru Saito, Kunihiko Tsuchiya, Hiroshi Kawamura et al., Density dependence on thermal properties of $\mathrm{Li}_{2} \mathrm{TiO}_{3}$ pellets. J. Nucl. Mater. 253, 213-218 (1998)

7. Pu Liu Songlin, Cheng Xiaoman Yong et al., Conceptual design of a water cooled breeder blanket for CFETR. Fusion Energ. Des. 89, 1380-1385 (2014)

8. A. Abou, A. Ying, M. Abdou, Effective thermal conductivity of lithium ceramic pebble beds for fusion blankets: a review. Fusion Sci. Technol. 47, 1094-1100 (2005)

9. Y. Someya, A feasible DEMO blanket concept based on water cooled solid breeder, In 24th IAEA Fusion Energy Conference, San Diego, USA (2012)

10. Liu Songlin, Li Jiangang, Zheng Shanliang et al., Neutronics analysis of inboard shielding capacity for a DEMO fusion reactor CFETR. Fusion Energ. Des. 88, 2404-2407 (2013)

11. Wang Ruijin, Zhang Kai, Wang Gang, Fluent technology foundation and application cases (Tsinghua University Press, Beijing, 2007), pp. 50-58. (in Chinese)

12. Lili Tong, Mufei Wang, Xuewu Cao et al., Preliminary analysis of in-vessel first wall cooling pipe ruptures for ITER. J. Fusion Energ. 34, 29-35 (2014)

13. Cheng X, Xuebin Ma, Jiang $\mathrm{K}$ et al. Thermal hydraulic design and analysis of water cooled ceramic breeder blanket for CFETR. Plasma Sci. Technol. (2015), (in press) 